\title{
Morphological and Mechanical Investigation of Double-Layer Reciprocal Structures
}

\author{
Cyril Douthe $\cdot$ Olivier Baverel
}

\begin{abstract}
The simple connection conditions of reciprocal structures means that technological constraints become geometrical constraints and bending moments are increased. Geometrical constraints can be dealt by using form-finding methods such as a dynamic relaxation algorithm, but resisting bending moments to gain stiffness is difficult to accomplish without increasing the weight of the structure. For standard reticulated structures, common strategies consist in introducing curvature in the structure and/or modifying the structure into a double-layer space structure. The proposed paper is thus an attempt to apply these strategies to reciprocal structures and to develop spherical domes with a structural thickness. Several configurations will be investigated and compared in term of geometrical feasibility and structural performance.
\end{abstract}

Keywords Reciprocal structures - Structural systems - Design analysis · Structural engineering · Morphology · Spatial structures · Form-finding · Dynamic relaxation $\cdot$ Linear elastic behaviour

\section{Introduction}

From a technological point of view, reciprocal structures have the advantage of simplifying considerably connections in the sense that members are connected only

C. Douthe $(\bowtie)$

Université Paris-Est, IFSTTAR, Cité Descartes 14-20 Bd Newton,

Champs-sur-Marne 77447, Marne-la-vallée Cedex 2, France

e-mail: cyril.douthe@ifsttar.fr

O. Baverel

Université Paris-Est, Laboratoire Navier (UMR 8205), IFSTTAR, ENPC, CNRS,

Cité Descartes 6-8 av Pascal, Champs-sur-Marne 77455, Marne-la-vallée Cedex 2, France

e-mail: olivier.baverel@enpc.fr 
by pairs. This constructional advantage has two main consequences: technological constrains are replaced by geometrical constrains and bending moments are increased through the non convergence of members at nodes with multiple connections. Indeed, elsewhere (Douthe and Baverel 2009) we have shown that the reciprocal structures are softer and generate higher stresses than their conventional counterparts. In order to increase the performances of reciprocal structures, here we investigate two basic strategies used for reticulated systems: the introduction of curvature and/or an increase in the structural thickness. Various configurations leading towards double-layer systems will be presented. In "Double-Layer Systems with Truss Elements", simple configurations where structural thickness is obtained by the elements themselves (Vierendeel trusses or any other space trusses) will be examined and a simple construction made of ladder elements will be shown. Then, in "Double-Layer Systems with Slender Members for Flat Configurations", a solution for building a double-layer reciprocal system with a flat configuration is developed based on analytical calculations (Sénéchal et al. 2011). In "Double-Layer Systems with Slender Members for Curved Configurations", a double-layer dome configuration is investigated. The form-finding method using the dynamic relaxation algorithm (Douthe and Baverel 2009) is detailed and a comparison of the structural behaviour with a single-layer configuration is made. Finally, we conclude with a discussion of the potential of double-layer reciprocal systems.

\section{Double-Layer Systems with Truss Elements}

A simple possibility for creating reciprocal systems with large span is to increase the structural thickness of each member individually in standard single-layer configurations similar to those shown in (Baverel et al. 2000) and (Baverel et al. 2004). For example, a beam element can be replaced by a truss element as in the structure shown in Fig. 1, which is made of ladders or Vierendeel trusses and was built on a scheme of linear elements to investigate the geometrical problems arising when the thickness of members is increased.

Connections are simply realised by laying and slightly overlapping members one on another reciprocally, sub-element on sub-element. Indeed in Fig. 2, which represents a characteristic reciprocal node (often called a fan), it can be seen that the upper and lower chords of each truss lie on top of each other reciprocally. This is made possible by rotating the trusses around their axes. Thus the trusses do not stand vertically, but are slightly tilted, with the degree of tilt depending on the height of the truss and the dimension of the sub-element. It must be noticed that the practical disposition for connections using sub-elements is specific to the kind of member used here and would have to be adapted for different kind of members.

Though only built for morphological purposes and not with any mechanical intention, it is worth noting that this structure has a span greater than $10 \mathrm{~m}$ and supports its own weight, which already proves its potential for practical applications. To go further, in (Douthe and Baverel 2009) and (Baverel 2000) it was demonstrated that, when loaded, the fans have a slight rotation due to the nonconvergence of members' axial forces toward the fan centre. This rotation generates 


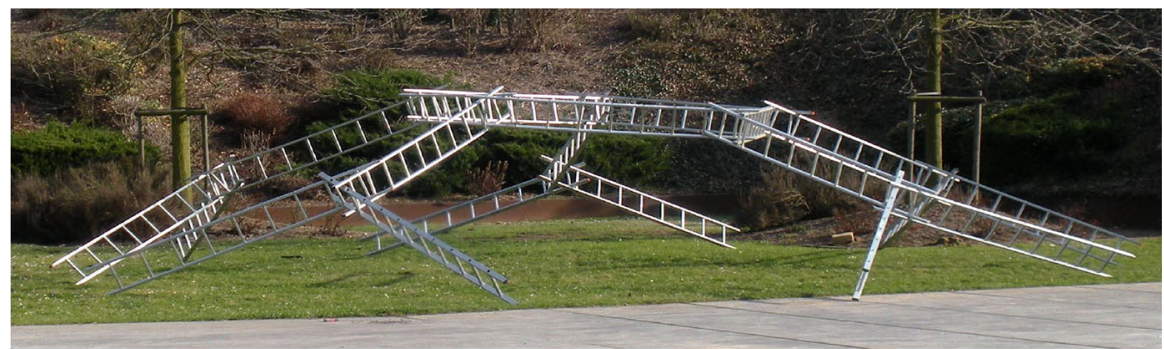

Fig. 1 Side view of a reciprocal dome with truss elements

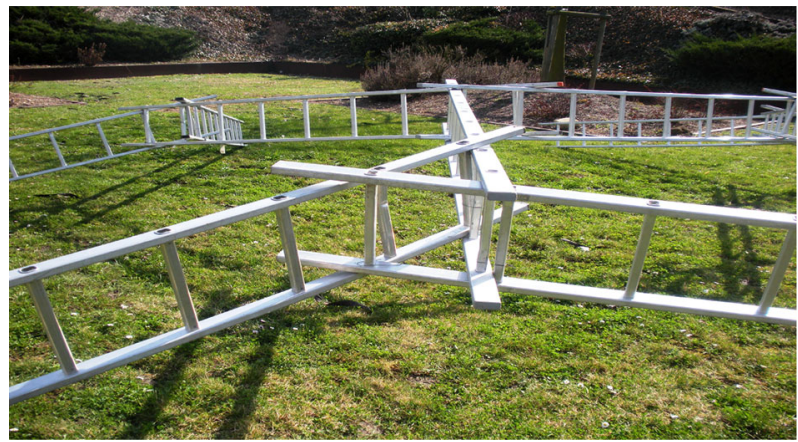

Fig. 2 View of a fan composed with three truss elements

moments along the weak axis of the members. In the present case, moments along the weak axes might have some significant effects on the overall stiffness of the structure; thus from a structural point of view, the choice of Vierendeel trusses is not optimal. Further investigation of the mechanical behaviour should thus consider using members made of spatial trusses. However, for such members, the degree of freedom in rotation around their axis of the Vierendeel trusses will be likely lost and the connection between members will be more complicated. We can see then that there is a compromise to be found between ease of construction and efficiency.

\section{Double-Layer Systems with Slender Members for Flat Configurations}

In "Double-Layer Systems with Truss Elements", the method proposed to obtain a "double-layer" structure was to start from a single-layer reciprocal structure and to replace slender members by space trusses. In the second method presented here, the double-layer reciprocal system is obtained by transformation of a standard doublelayer spatial structure in a similar way to the transformations studied in (Sénéchal et al. 2011; Baverel et al. 2004; Baverel and Nooshin 2007). There are several way to compute practically the final configuration of the reciprocal system after the transformation: some analytical methods have been developed by rotation of members in (Sénéchal et al. 2011) and (Baverel and Nooshin 2007) and also some 
numerical methods using genetic algorithm (Baverel et al. 2004) or dynamic relaxation (Douthe and Baverel 2009). Here we will focus on the analytical method, while "Double-Layer Systems with Slender Members for Curved Configurations" will make use of the dynamic relaxation algorithm.

The analytical developments presented in the following concentrate on one specific configuration shown in Fig. 3 (Bangash and Bangash 2003) which consists in a two-way space grid with square pyramids. When transformed into a reciprocal system, the members are rotated so that multiple connections disappear and members are only connected by pairs. Considering the symmetries of the spatial structure, all nodes and all fans are identical (except, of course, for the fans lying on the perimeter of the spatial frame). The configuration of a typical fan of the upper layer after transformation is shown in Fig. 4, where members laying on cups represents the members that are on the upper layer and those laying on the table represent the diagonal elements that connect upper and lower layer in Fig. 3. Fans of the lower layer are obtained by simply mirroring fans of the upper layer.

The parameters of the model illustrated in Fig. 4 are: the length and engagement length of the members of the upper or lower layer (i.e., the distance between connections on a member end); the length and engagement length of the diagonal members; and the eccentricity of the connection (or the distance between the axes of two connected members). There are thus five design parameters. The strategy for determining analytically the values of these parameters is the same as in (Sénéchal et al. 2011):

- Write that the direction of the eccentricity between two members is perpendicular to each member,

- Write that the distance between two members is equal to the eccentricity,

- Write that the distance between two contact points on a member is equal to the engagement length.

- Write geometric compatibility between upper and lower fan (which is equivalent to adjusting the diagonal length so that upper and lower fans can effectively be connected).

To facilitate calculations [which are detailed in (Sénéchal 2006)], it is presumed that the relative engagement length are equal and that the eccentricity is constant. Given those conditions, we find that, out of five parameters, only two are
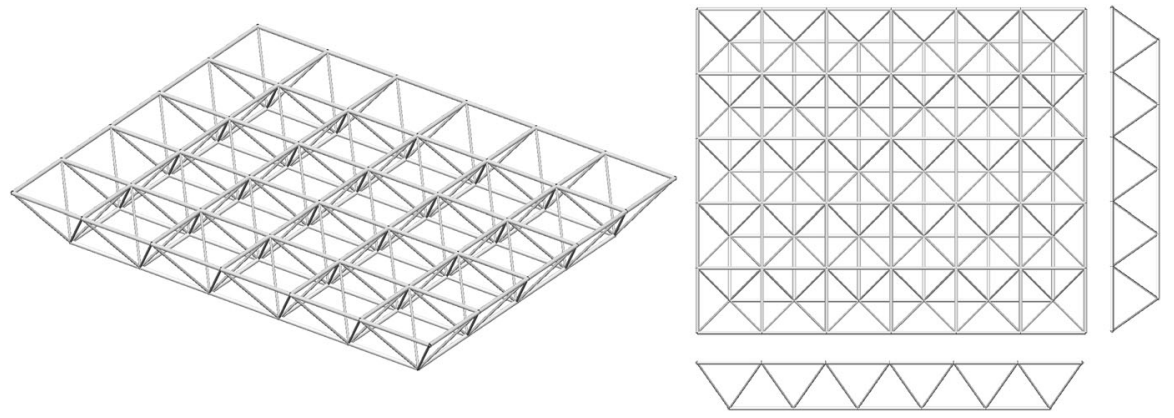

Fig. 3 Two way space grid with square pyramid (square on square offset) 


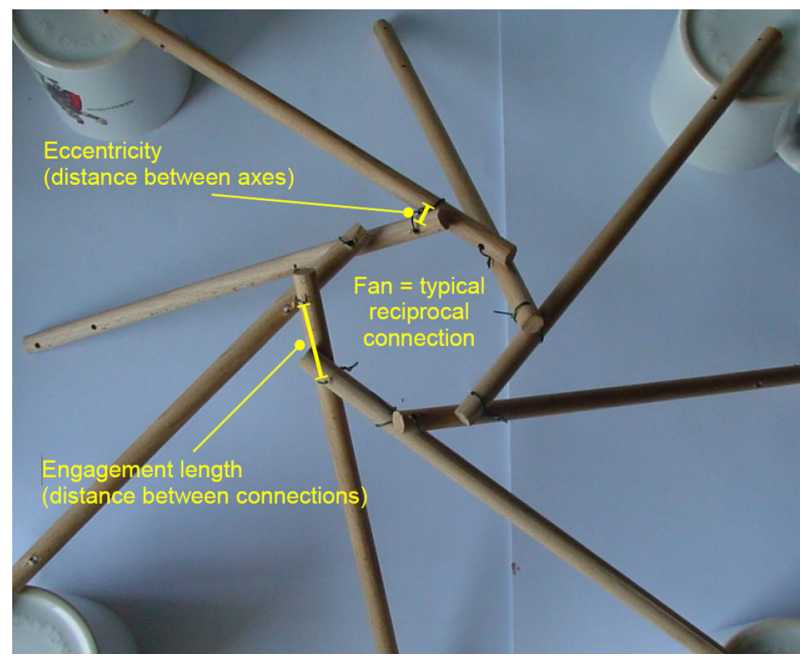

Fig. 4 Perspective view of a fan

independent: the length of the upper (or lower) members and the eccentricity. Neglecting homothetic configurations, the geometry of the basic cell of the reciprocal system, shown in Fig. 5, is thus entirely determined by the ratio of the eccentricity to the length of a member. In particular, the height of the double-layer system which is a key parameter for the mechanical behaviour, is given by that ratio: the higher the ratio of eccentricity to length, the higher the ratio of height to length. Introducing some practical data by taking a member length of $100 \mathrm{~cm}$ with an eccentricity of $4 \mathrm{~cm}$, we find that the necessary engagement length is close to $3.6 \mathrm{~cm}$ and the height of the structure of $107 \mathrm{~cm}$ (see Fig. 5).

Extending this configuration over several fans, we obtain the reciprocal system shown in Fig. 6. It is higher and denser than the initial configuration shown in Fig. 3. As it is, the reciprocal structure is thus relatively difficult to build (engagement length and eccentricity being of the same order of magnitude) and also less efficient from a mechanical point of view as it uses more material for the diagonals, which contribute very little to the overall stiffness. The only solution for improving the mechanical behaviour of this double-layer reciprocal system is thus to abandon the hypothesis of equal relative engagement length of members and diagonals, so that the height becomes again an independent design parameter. This, however, will add one more parameter in a complex non-linear analytical problem which is still under investigation.

\section{Double-Layer Systems with Slender Members for Curved Configurations}

Form-Finding of Double-Layer Grids

The configuration investigated in this section is inspired by a scheme already illustrated by R. Buckminster Fuller for spatial structures, which he had called 

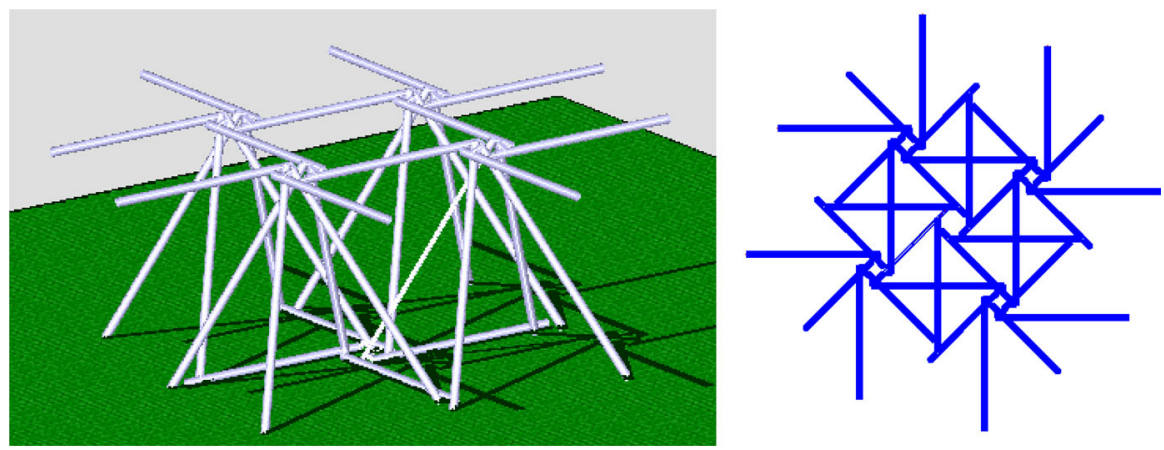

Fig. 5 Axonometric and plan view of a configuration with five fans

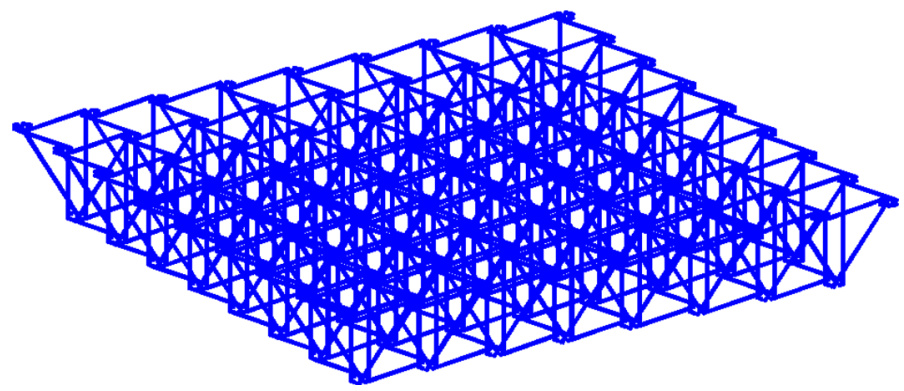

Fig. 6 Plane double-layer reciprocal system with 81 fans

"magnesium-framed geodesic domes". ${ }^{1}$ The idea is to connect two superimposed triangular grids with two different lengths (the larger one being $\sqrt{ } 3$ times longer than the other), as shown in Fig. 7. The nodes of the larger grid coincide with nodes of the smaller grid, so that a total number of twelve members converge at these nodes, which form the upper layer of the spatial systems. The nodes of the smaller grid that do not coincide with nodes of the larger grid are connected directly with each other through an hexagonal mesh with nodes to which six members converge. These nodes form the lower layer of the spatial systems. The members connecting the lower hexagonal mesh to the upper triangular mesh insure the transfer of shear forces between the two layers. These three types of member (upper layer, lower layer and diagonals) are visible on Fig. 7. The objective here is thus to transform this flat grid, in which multiple members converge at nodes, into a double-layer reciprocal system.

In "Double-Layer Systems with Slender Members for Flat Configurations", the system was extremely symmetric and the irregularities of the system at boundaries were neglected, so that an analytic solution for the transformation could be found. This time, symmetries are more complex and practical solutions for the interruption

\footnotetext{
1 An image of one such structure is shown on the webpage http://designmuseum.org/design/rbuckminster-fuller.
} 
of the grid at the boundary are desired. The transformation into a reciprocal system will thus be done numerically with a form-finding method based on the dynamic relaxation algorithm (Douthe and Baverel 2009). The principle of this method relies on the introduction of a fictitious mechanical problem where members initially converging at nodes are bent to form a reciprocal system. The solution of the problem is a structure in equilibrium where the bending energy of all members has been dissipated and where members are straight. The fictitious mechanical properties are defined according to the designer's needs as will be illustrated in "Form-Finding of a Single-Layer Equivalent Grid". Then, as in the final configuration members are straight, there is no need to finely model the curvature. Convergence speed is thus increased by using the smallest number of nodes required to describe the members' geometry, which is only four nodes per member (one node at each connection). Convergence is also improved by the use of a routine which has been added to the method presented in (Douthe and Baverel 2009) and which consists in gradually increasing the bending stiffness of members and updating their reference length as they straighten.

The geometrical parameters of the form-finding procedure consist in a set of two configurations: a reference configuration which is stress free and used to evaluate at each time step the stresses in the members, and an initial configuration from which calculations are started. Since by definition the members are straight, the reference configuration reduces to a set of reference lengths of the members. The reference length for the members of the hexagonal grid is thus set to $100 \mathrm{~cm}$ with an engagement length of $10 \mathrm{~cm}$, that of the members of the larger triangular grid is consequently $173.2 \mathrm{~cm}$, while their engagement lengths are the same, $10 \mathrm{~cm}$. Then, since the intermediate members connecting the upper and lower grid will be used as spacers between the two layers, their length is set as $122.1 \mathrm{~cm}(100 \mathrm{~cm}$ in projection on the grids planes and $70 \mathrm{~cm}$ vertically); thus, after form-finding, the structural height should be close to $70 \mathrm{~cm}$.

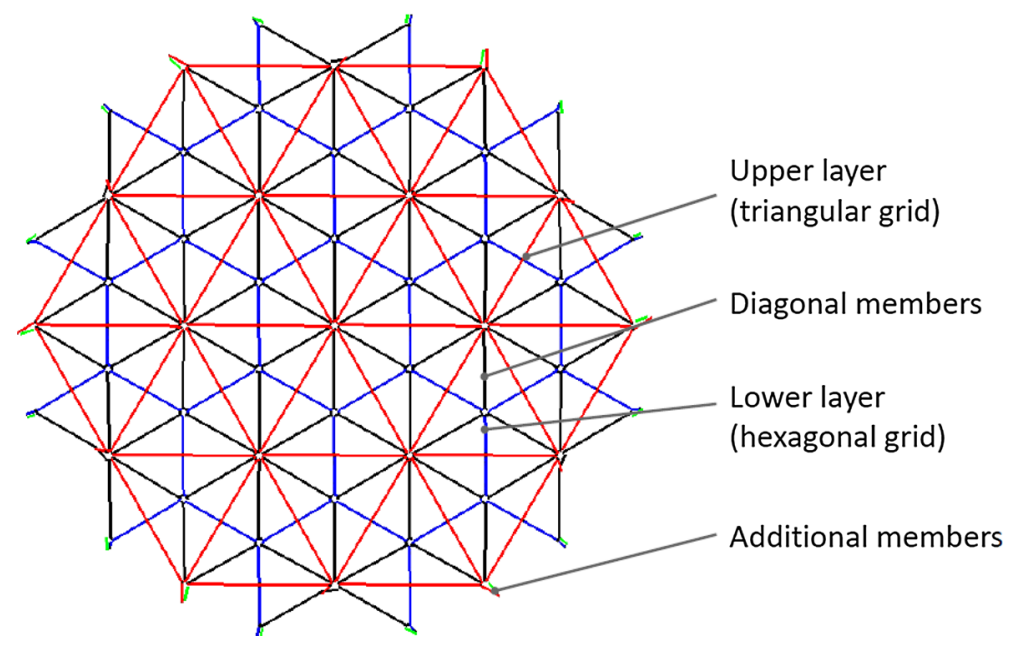

Fig. 7 Principle scheme of the double-layer grid 
At the external nodes located on the boundary of the grid, the regularity of the reciprocal system is necessarily interrupted: the number of members converging at nodes is different from nodes in the interior part of the grid. To maintain a certain regularity for these external fans, some adjustments have been made by locally modifying the engagement length of a member or by the use of additional members of suitable lengths (see the locations of the modifications in Fig. 7). An example of each adjustment is illustrated in Fig. 8. Figure 8a shows an external fan to which nine members are converging instead of the expected twelve and where it is necessary to increase the engagement length of one member to close the truncated dodecagon. Figure $8 \mathrm{~b}$ then shows an external fan into which a new member has been added. Initially five members (three members from the upper layer and two interconnecting members) converge toward this external node which would have a dodecagonal form if located in the interior part of the grid. A sixth member with the appropriate length is thus added to close the open polygon respecting the geometry of the dodecagon. This construction principle is applied to all incomplete hexagons and dodecagons of the grid of Fig. 7, so that in total eighteen additional members are introduced and twelve engagement lengths modified.

For the initial configuration, the choice of lengths is up to the designer, provided that geometrical constraints are satisfied, that boundary conditions and connectivity of nodes are respected. The initial configuration is thus chosen to guide the formfinding toward a solution that suits the designer's needs. Here, the initial configuration is defined by projecting the grid of Fig. 7 on a sphere with a $5 \mathrm{~m}$ radius and by bending the members' extremities to satisfy the reciprocal system connectivity with a constant eccentricity for all connexions of $4 \mathrm{~cm}$ (see Fig. 9). To achieve this transformation of the initial grid into a reciprocal structure by bending, it is necessary to define how the members initially converging at the same node will rely on each other in the reciprocal structure. The transformation of each node is characterised by two parameters which elsewhere have been called [in (Baverel et al. 2000)] the 'disposition' and the 'style' of the fan. Here the dispositions of fans are chosen so that, after transformation, fans of the lower layer form downward fans (with eccentricities pointing along the inward normal of the surface) while the fans of the upper layer form upward fans (with eccentricities pointing along the outward normal of the surface); see the enlarged details in Fig. 9. Both layers can thus be easily connected. Then, in (Douthe and Baverel 2009) we had remarked that, from a mechanical point of view, alternating fan styles significantly increases the rotations of the fans and the bending moments in the members. All fans are thus defined with the same style: rightward.

Finally the fictitious mechanical properties of the members are set so that their bending stiffness is much larger than their axial stiffness, so that the lengths of members are susceptible of finite variations. They can hence adapt to the various geometrical constraints and still stay straight (the out-of straightness after formfinding should be $<10^{-3}$ ).

Given all these parameters, the result of the form-finding or the final configuration is shown in Fig. 10. The two grids (the lower hexagonal and the upper triangular) lay on two different pseudo-spheres with a radius of about $6.0 \mathrm{~m}$. The distance between the layers is approximately $70 \mathrm{~cm}$ as expected. During the 
a

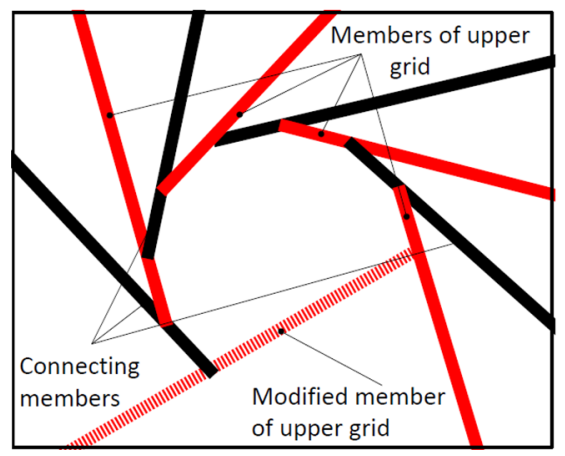

b

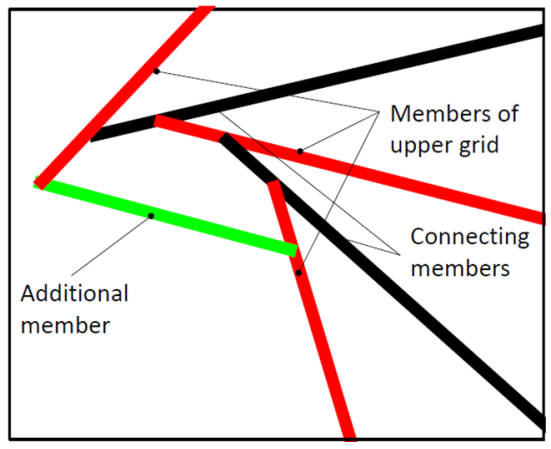

Fig. 8 Adjustment of external fans (a, left), engagement length (b, right) additional member

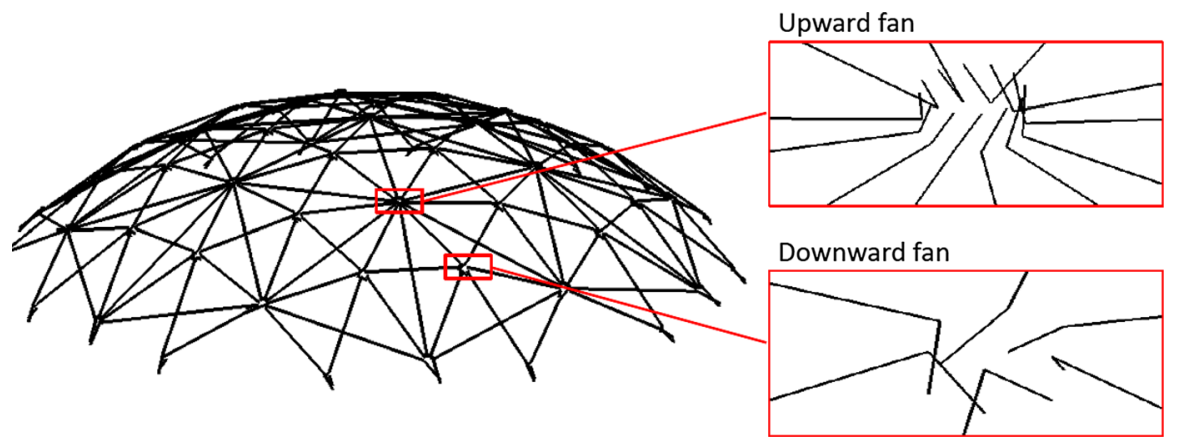

Fig. 9 Initial configuration with bent members

form-finding, the lengths of the members have changed slightly. The members of the larger grid (forming the upper layer) have an average length of $183.0 \mathrm{~cm}$ with an engagement length around $10.5 \mathrm{~cm}$. Those of the hexagonal grid have a length of $103.5 \mathrm{~cm}$ with an engagement length of $9.5 \mathrm{~cm}$. The length of the interconnecting members is also around $123 \mathrm{~cm}$, but their engagement lengths vary depending on the layer of the extremity: around $6.0 \mathrm{~cm}$ in the upper layer and $17.5 \mathrm{~cm}$ in the lower layer. These lengths are slightly different at the extremities of the grid, where the regularity of the scheme is only partly conserved.

Considering the number of parameters that enter into the form-finding procedure (namely, the geometry of members, the disposition and style of the fans, the initial configuration and the boundary conditions), the reciprocal double-layer structure shown in Fig. 10 is only one among an infinity of possible structures. At this stage, where only geometrical issues have been discussed, the criterion for choosing a suitable structure is constructability. Practical experience from previous experiments with regular polyhedra (Sénéchal et al. 2011; Baverel and Nooshin 2007) have proved that engagement lengths larger than 1.5 times the eccentricity between members axes are sufficient to insure feasibility and placement of connectors. As 

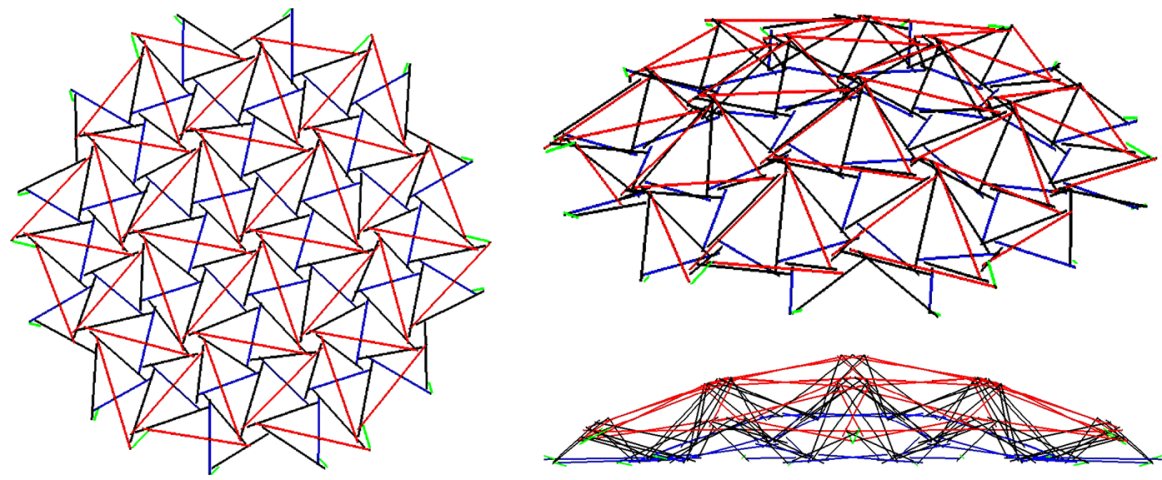

Fig. 10 Final configuration of the double-layer reciprocal system

this is the case here, the reciprocal structure seems suitable. Further optimisation should thus be made relying on the mechanical behaviour, which will be investigated in section "Mechanical Behaviour of the Two Configurations".

\section{Form-Finding of a Single-Layer Equivalent Grid}

An equivalent single-layer grid is obtained by transformation into a reciprocal structure of the smaller triangular grid shown in Fig. 7 with no additional members on the boundary. The geometrical parameters of the form-finding procedure are thus the following: an identical reference length of $100 \mathrm{~cm}$ with an engagement length of $20 \mathrm{~cm}$ (two times larger than previously because the number of members converging at a node is two times smaller) and an eccentricity of $4 \mathrm{~cm}$. All fans have upward disposition and rightward style. The initial configuration of the grid is such that the nodes lay on a spherical dome with a radius of $5 \mathrm{~m}$. It must be specified here that this time the position of all external nodes is fixed. As previously, the fictitious mechanical properties of the members are set so that their bending stiffness is much larger than their axial stiffness; their lengths are thus adaptable. The result of the form-finding is shown in Fig. 11.

In the final configuration of Fig. 11, the reciprocal structure has a height of $1.94 \mathrm{~m}$ for a $7 \mathrm{~m}$ span which corresponds to a spherical dome with a radius of $4.1 \mathrm{~m}$. As for the double-layer structure, during the form-finding, the lengths of the members have changed and vary between $143 \mathrm{~cm}$ at the crown to $116.5 \mathrm{~cm}$ close to the supports. The engagement lengths vary between $34.5 \mathrm{~cm}$ at crown to $21.5 \mathrm{~cm}$ close to the supports. This dome structure is indubitably constructible, and due to its double-curved shape, should prove to have good mechanical behaviour despite a relatively large engagement length.

\section{Mechanical Behaviour of the Two Configurations}

During the form-finding step, fictitious mechanical properties had been used to find the geometry of the previous reciprocal systems. In the geometries shown in Fig. 10 

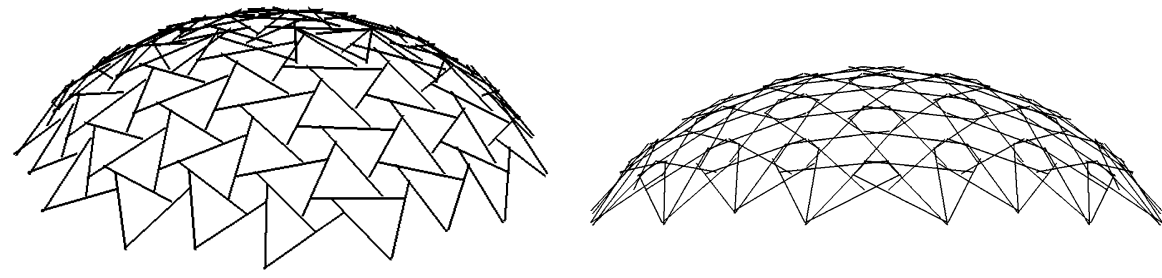

Fig. 11 Equivalent single-layer reciprocal structure

or 11 , the members are straight but stretched (elongated) because initially the lengths of the members are unknown. Before conducting any mechanical analyses under external load, it is thus necessary to relieve the systems from inner axial stresses induced by these elongations. This is done by setting each member's length at rest (the reference length) equal to its length in the final configuration obtained by form-finding. The removing of these stresses in either the single-layer or doublelayer structure has no effect on the geometry of the structure (or, more precisely, the changes in the form are smaller than the out-of-straightness tolerated in the formfinding).

Then, for the study of the mechanical behaviour, realistic mechanical and geometrical properties are introduced. Members are thus taken to be steel circular hollow sections with an external diameter of $40 \mathrm{~mm}$ and a thickness of $3 \mathrm{~mm}$. Boundary conditions consist in blocking the positions of nodes located on the periphery of both structures. For the single-layer dome, the boundary conditions are thus identical to those used for the form-finding. For the double-layer structure, it is not easy to precisely define external nodes considering the number of members touching the periphery, so that it was chosen to support the structure on the additional members shown in Fig. 8, whose six degrees of freedom have been fixed.

Concerning external loads, two cases have been investigated: one symmetric, uniformly-distributed load and one asymmetrical distributed load on half of the structure. To ease comparison between systems, these distributed loads have been introduced via vertical point loads applied at each end of every member of the smaller grid (i.e., every member of the single-layer system and only the shorter members of the double-layer system). The intensity of each point load is $0.5 \mathrm{kN}$, which corresponds approximately to $3.5 \mathrm{kN} / \mathrm{m}^{2}$ (an acceptable density for a roof structure).

From a numerical point of view, non-linear analyses are conducted using the same algorithm as for the form-finding procedure (reproducing the methodology used in [Douthe and Baverel 2009)]. Convergence is reached when the initial kinetic energy of the structure has been dissipated (practically when the current kinetic energy peak is below 1/100th of the initial/maximum peak). It must be noted here that the poor meshing of the form-finding has been slightly improved for the accuracy of results and that the density of the mesh has been refined to reach six nodes per member: one for each end connection, one for each engagement connection and then two intermediate nodes. Mechanical behaviour is assessed through: the overall stiffness of the structure (measured through the average and 
maximal displacement of the loaded points and the rotation of the fans), the intensity of reactions and inner stresses in the members (essentially axial forces and bending moments).

Table 1 shows the displacements under the two loadings. We first notice that under both loadings the displacements are low and would satisfy typical slope criterion for roofing structures: here 1/200th of the span is equal to $35 \mathrm{~mm}$. We also observe that, under asymmetric loading, the maximum displacements are of the same magnitude than under symmetric loading, denoting the very same mechanical behaviour. Comparing both structures, we see that the displacements in the singlelayer structure are greater than those of the double-layer structure, which shows that the system effectively has a significant structural height. Moreover, we remark that fan rotations (which are caused by the presence of eccentric normal forces converging at the fan) are of very low magnitudes, especially in the double-layer system. This difference between the two systems is related to the fact that the singlelayer structure works mainly as a membrane structure with forces that flow within the surface, whereas the double-layer structure works as a thick plate, where forces flow within the thickness of the plate. That being said, the order of magnitude of the rotations in the single-layer dome are comparable to those observed in the dome of (Douthe and Baverel 2009) (taking into account the fact that here the Young modulus is twice as large, one finds $1.2^{\circ}$ in (Douthe and Baverel 2009) and $0.5^{\circ}$ here), which confirms that the influence of the fan rotations on the general behaviour can be considered as a second-order effect.

Table 2 shows the extreme values of the forces in both structures. As expected, we observe that the horizontal thrust relatively to the vertical reaction is higher in the single-layer structure and that the inclination of the total reaction is approximately that of the last member. This confirms that the single-layer system carries load mainly within its surface, and that its behaviour is dominated by membrane actions. These membrane actions correspond to normal forces in the members of the reciprocal systems and, as these forces are not converging toward the same points, they create high bending stresses. Their values, however, remain such that the construction of the structure seems feasible, either by using a steel grade of S355 or by slightly increasing the inertia of the members. The distributions of the normal forces under symmetric and asymmetric loading can be seen,

Table 1 Comparison of displacements of single and double-layer reciprocal systems

\begin{tabular}{llllll}
\hline & \multicolumn{2}{l}{ Symmetric loading } & & \multicolumn{2}{l}{ Asymmetric loading } \\
& Average & Maximum & & Average & Maximum \\
\hline Vertical displ. (mm) & 7.5 & 11.5 & & 3.0 & 6.0 \\
Double-layer & 12 & 30 & & 5.5 & 29 \\
Single-layer & & & & 0.1 \\
Fan rotation (deg) & $\approx 0.0$ & $\approx 0.1$ & & $\approx 0.0$ & 0.5 \\
Double-layer & 0.2 & 0.5 & & $\approx 0.0$ & 0.0 \\
Single-layer & & & & \\
\hline
\end{tabular}


Table 2 Comparative of forces in single and double-layer reciprocal systems

\begin{tabular}{lccccccc}
\hline & $\begin{array}{l}\text { Max } \mathrm{R}_{\mathrm{H}} \\
(\mathrm{kN})\end{array}$ & $\begin{array}{l}\mathrm{Max} \mathrm{R}_{\mathrm{V}} \\
(\mathrm{kN})\end{array}$ & $\begin{array}{l}\mathrm{N}_{\min } \\
(\mathrm{kN})\end{array}$ & $\begin{array}{l}\mathrm{N}_{\max } \\
(\mathrm{kN})\end{array}$ & $\begin{array}{l}\mathrm{M}_{\max } \\
(\mathrm{kN} . \mathrm{m})\end{array}$ & $\begin{array}{l}\sigma_{\min } \\
(\mathrm{MPa})\end{array}$ & $\begin{array}{l}\sigma_{\max } \\
(\mathrm{MPa})\end{array}$ \\
\hline $\begin{array}{l}\text { Symmetric loading } \\
\text { Double-layer }\end{array}$ & 9.8 & 13.1 & -6.0 & 4.3 & 0.57 & -188 & 189 \\
$\begin{array}{l}\text { Single-layer } \\
\text { Asymmetric loading }\end{array}$ & 6.0 & 8.0 & -7.2 & 2.2 & 0.71 & -245 & 228 \\
$\begin{array}{l}\text { Double-layer } \\
\text { Single-layer }\end{array}$ & 9.4 & 14.4 & -9.5 & 9.0 & 0.59 & -194 & 199 \\
\hline
\end{tabular}
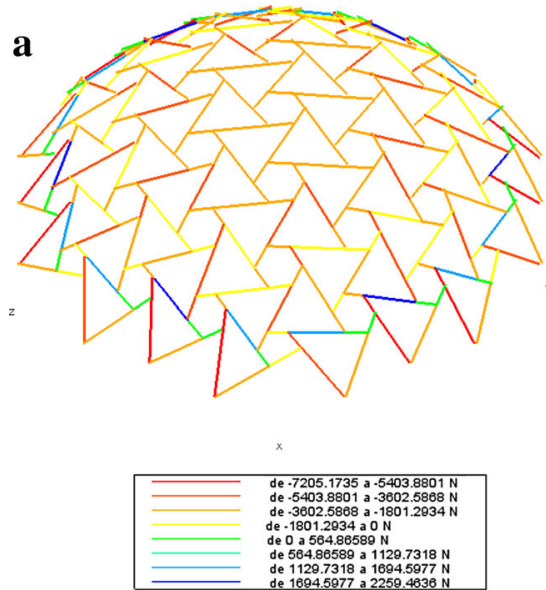
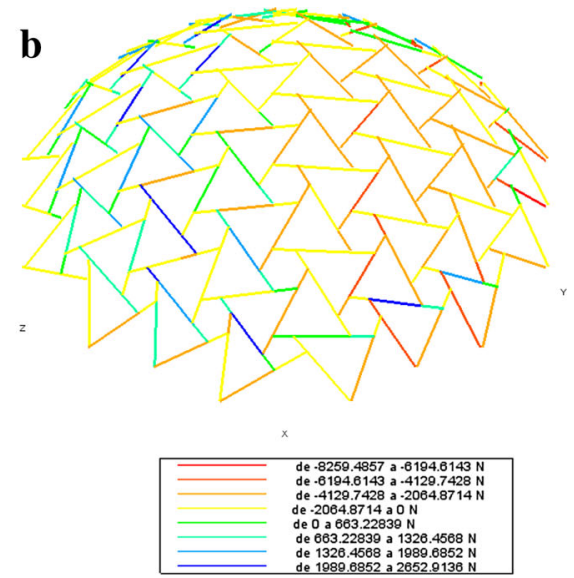

Fig. 12 Normal forces in the single-layer dome. a, (left) under symmetric loading; b, (right) under asymmetric loading

respectively in Fig. 12a, b (in which the left half of the structure is the loaded one). In both loading cases, the magnitude of forces are comparable. Under symmetric loading, all members are in compression, except for a kind of tension ring which overtakes hoop forces above supports as would be the case in a spherical shell. Under asymmetric loading, compression dominates the loaded half of the structure while a more complex distribution of tension and compression is observed in the other half.

Concerning the bending moments, their distributions are shown in Fig. 13. In both loading cases, we see that they develop mainly in the loaded areas and that the closer the members are to the supports, the lower the magnitudes. However, we also notice that, despite a lower total load under asymmetric loading, the highest value of the bending moment is $15 \%$ higher than under symmetric loading. Once again, this is a phenomenon that can be observed in continuous spherical shells, in which asymmetric loadings often generate higher stresses than symmetric ones. 

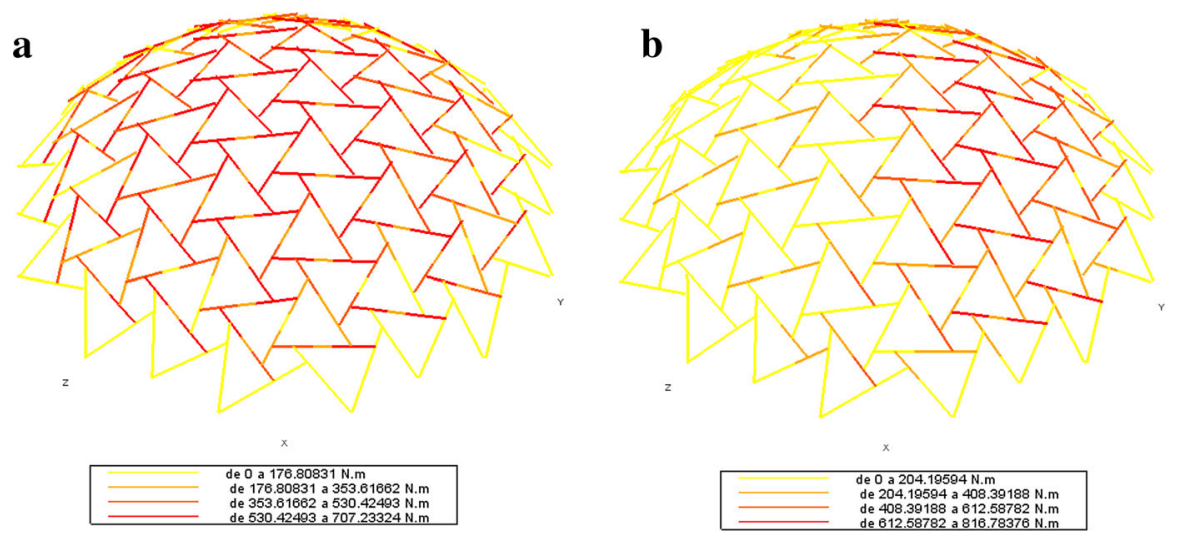

Fig. 13 Bending moments in the single-layer dome. a, (left) under symmetric loading; b, (right), under asymmetric loading
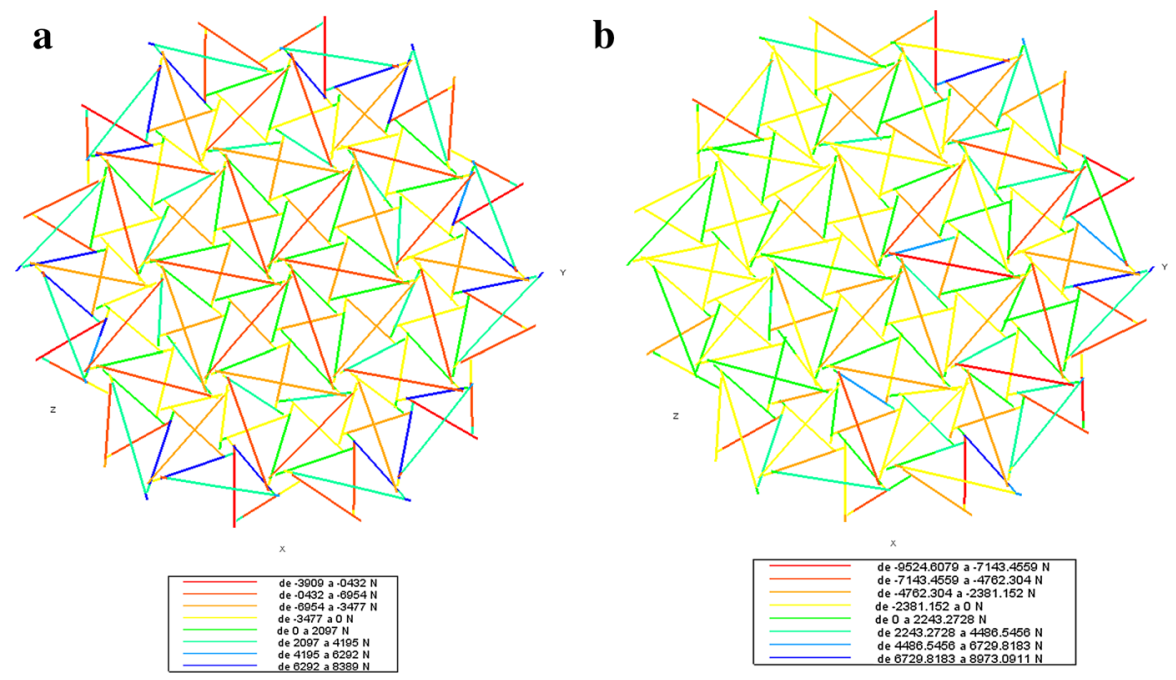

Fig. 14 Normal forces in the double-layer structure. a, (left) under symmetric loading; b, (right) under asymmetric loading

The general behaviour of the double-layer system is quite different as it is globally dominated by a thick shell behaviour and bending effects. The loads acting perpendicular to the surface are resisted through compression in the upper layer (which consists in a triangular grid) and tension in the lower layer with some compression, as it consists in an hexagonal grid. This is illustrated in Fig. 14, where hot colours correspond to compression. Moreover, Table 2 shows that compression forces are approximately of the same magnitude in the double-layer structure as in the single-layer dome, but that tension forces are greater. This is related to the fact that, this time, tension forces do not correspond to hoop action but to shear forces within the shell, which are logically greater at the supports. 

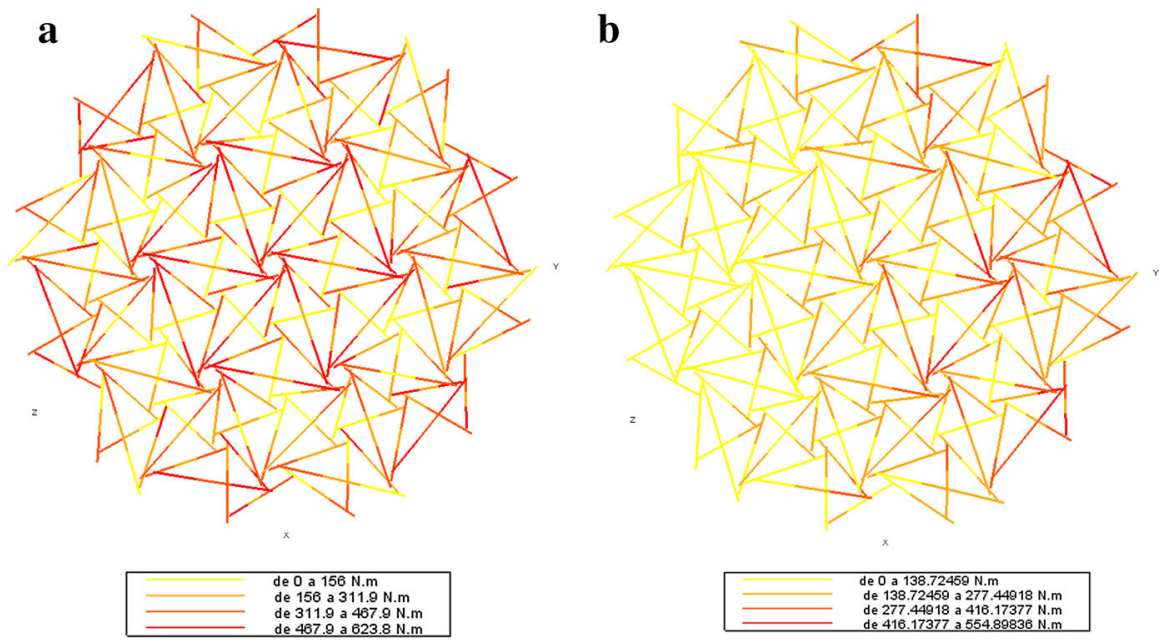

Fig. 15 Bending moments in the double-layer structure: a, (left) under symmetric loading; b, (right) under asymmetric loading

An another illustration of the fact that the reciprocal system effectively works like a double-layer thick shell can be found when comparing the magnitude of forces and bending moments under symmetric and asymmetric loading (Fig. 15): they are almost equal, the double-layer system resists in the same way to both loadings in a way that is typical of structures that carry loads by bending.

\section{Conclusions}

Different configurations to improve the efficiency of reciprocal systems have been investigated in this paper. The first configuration showed the potential of using spatial members to create reciprocal configurations with structural thickness. The second configuration then illustrated how planar double-layer reciprocal systems can be obtained through the transformation of standard spatial grids. The results of analytical developments on a two-way square grid have been presented. However the too-restrictive hypotheses made for the analytical calculations of the geometry led to engagement lengths that are quite small compared to the diameter of the members, thus making construction difficult. Further development with more design parameters are thus currently under progress.

In the final section, two spatial configurations of reciprocal systems were investigated. The first one is a double-layer dome based on the superposition of two triangular grids, the second one a single-layer dome based on a triangular grid. A form-finding method for the definition of geometries compatible with reciprocal arrangement of members was illustrated. Then a mechanical study of the two structures was conducted. On the one hand, the double-layer reciprocal structure works effectively like a spatial structure, like a thick plate in bending with high 
bending stiffness and low sensitivity to asymmetric loading; on the other hand, the single-layer dome works like a thin shell dominated by membrane forces and is therefore more sensitive to asymmetric loading. However, we have seen that both structures are efficient and capable of satisfying standard design criteria for roofing structures.

The structural typologies which can be achieved with reciprocal systems seem thus to be as varied as those obtained with standard typologies. This means that, for designers, there is thus a very wide universe of morphologies to explore, and ample room for optimisation and creativity.

\section{References}

Bangash, M.Y.H., and T. Bangash. 2003. Elements of spatial structures: analysis and design. London: Thomas Telford.

Baverel, O. 2000. Nexorades: a family of interwoven space structures. PhD. thesis, University of Surrey. Baverel, O., and H. Nooshin. 2007. Nexorades based on regular polyhedra. Nexus Network Journal 9(2): 281-299.

Baverel, O., H. Nooshin, and Y. Kuroiwa. 2004. Configuration processing of nexorades using genetic algorithms. Journal of the IASS 45(2): 99-108.

Baverel, O., H. Nooshin, Y. Kuroiwa, and G.A.R. Parke. 2000. Nexorades. International Journal of Space Structure 15(2): 155-159.

Douthe, C., and O. Baverel. 2009. Design of nexorades or reciprocal frame systems with the dynamic relaxation method. Computer and structures 87: 1296-1307.

Sénéchal, B. 2006. Nexorades, analyses géométriques et constructions. Master's thesis, École Nationale des Ponts et Chaussées (in French).

Sénéchal, B., C. Douthe, and O. Baverel. 2011. Analytical investigations on elementary nexorades. International Journal of Space Structures 26(4): 313-320.

Cyril Douthe is a researcher at the Department of Material and Structural Engineering of the IFSTTAR Institute, Université Paris-Est, and a lecturer at the Ecole des Ponts ParisTech. His research activities cover aspects of behaviour, analysis and design of lightweight structures, with emphasis of problems of geometrically and material nonlinear behaviour, form-finding and structural optimization for elastic gridshells, reciprocal systems, steel arches, curved steel panels or rock fall barriers.

Olivier Baverel is Assistant Professor at the National School of Architecture in Grenoble (France), Assistant Professor at the Ecole des Ponts ParisTech, and a research fellow of the Laboratoire Navier (UMR ENPC/IFSTTAR/CNRS, France). He is also, since 2012, editor of the International Journal of Space Structures. 